\title{
Short-term Wind Power prediction Based on EEMD-Permutation Entropy and Elman
}

\author{
Xin-You Wang ${ }^{1, a}$ and Chen-Hua Wang ${ }^{2}$ \\ ${ }^{1}$ Institute of Technology, Gansu Radio \& TV University, Lanzhou 730030, China \\ ${ }^{2}$ Northwest Engineering Corporation Limited, PowerChina, Xi'an 710065, China \\ awangxiny@gsrtvu.cn
}

\begin{abstract}
In this paper, a new combination prediction approach was proposed and applied to improve the short-term wind power prediction precision. Firstly, the wind power sequences were decomposed into a limited number of sub-temporal sequences with different characteristics by using CEEMD-Permutation Entropy, which avoids the large computing scale problems of local analysis of wind power series. Then, the Elman prediction models were constructed by the feature analysis for each sub-temporal sequence respectively. And the final prediction values were given by the superposition of each sub sequences prediction. The approach is applied to Alberta, Canada wind farm forecasting. A comparison of our approach to existing prediction methods was also given. Simulation results show that the new model proposed in this paper can significantly improve the load prediction accuracy.
\end{abstract}

Keywords: Short-term wind power prediction; Complementary Ensemble Empirical Mode Decomposition (CEEMD); Permutation Entropy(PE); Elman Neural Network

\section{Introduction}

As the rapid development of wind power technology, the proportion of the wind turbine installed capacity increasing gradually in regional electrical system. However, large-scale wind power integration poses great challenges to both dispatching department of power system and the reliability of power supply system, due to the unstable output of wind power affected by the intermittent and volatility of wind. Thus, accurate and reliable wind power prediction has vital significance [1-2].

The wind power output performance as the volatility strongly nonlinear time series, resulting from the intermittent and volatility of wind power. At present, various computational intelligence for prediction such as ANN and SVM [3-5], which can describe the nonlinear relationship between the input and output of the wind power from its historical time series, have been widely used in the prediction of wine power. Since lack of the grasping for the changes of wind power itself, those single prediction methods are difficult to obtain high forecast precision substantially. Generally, large errors occur in the wind power prediction point that changed strongly.

In order to improve the wind power forecasting accuracy, the variation of the wind power forecasting should be found upon the analysis of the main factors that influence power prediction. Wind power sequences are kinds of nonstationary time series characterized by periodic components and random variation. In recent years, a new signal processing method, empirical mode decomposition (EMD), the technique allows for decomposing any complex signal into a collection of intrinsic mode functions (IMFs), so it is suitable to analyze instability and non-linearity time series. But, mode mixing occurred in EMD sometimes, and that makes the physical meaning of individual IMF unclear [6-8].

The Ensemble Empirical Mode Decomposition (EEMD) is a substantial improvement 
over the original EMD. It utilizes the full advantage of the statistical characteristics of white noise to perturb the signal in its true solution neighborhood, and effectively overcome the scale separation problem without introducing a subjective intermittence test in EMD[9-12]. However, with the help of added noises, EEMD essentially resolve the mode mixing problem associated with EMD, but the resulting IMFs derived from EEMD would inevitably be contaminated by the added noise especially when the number of ensemble is relatively low. This is especially true in the reconstruction of the signal from the IMF components, though this affects can be reduced by large scale means, it's much time-consuming. To improve the efficiency of the original noise assisted algorithm of EEMD, the complementary ensemble empirical mode decomposition (CEEMD) approach was proposed [NORDEN E.HUANG et al. (2010)]. Unlike the EEMD, CEEMMD uses each noise in pairs with plus and minus signs [13]. The advantage for this new approach, however, is to have an exact cancellation of the residual noise in the reconstruction of the signal with low ensemble numbers.

In Elman network, context units save previous outputs values of hidden layer neurons, and all signals from neuron outputs are connected as network inputs through unit time delay, and thus achieve a low resolution memory. Therefore, both the stability and the ability to deal with dynamics information have been improved [14].

On the basis of the preceding narrative, this paper proposes a model of wind power forecasting based on CEEED, Permutation Entropy and Elman neural network. Firstly, we applied the method of CEEED to decompose the wind power sequences into a number of stationary series and a certain trend in weight based on its characteristics. Then, the complexity of each IMF was measured by PE, and new sub-sequences were combined by the PE value, different Elman neural network model will be constructed and applied to the analysis of those sub-sequences. Finally, we will get the forecasting results by synthesizing the prediction of each component. The simulation results show that the method is of high precision and strong adaptability.

\section{EEMD and CEEMD}

\subsection{EEMD Theory}

The Ensemble Empirical Mode Decomposition (EEMD) [ZHAOHUA WU et al. (2009)] is a noise assisted method designed for eliminating the mode-mixing problem caused by intermittence signals in Empirical Mode Decomposition (EMD) method. To decompose the true signal of any complex data, this approach effectively resolve this problem by utilizing the full advantage of the statistical characteristics of white noise. That makes the signal continuous in different scales, and different scales of the signal region will be automatically corresponded to the suitable scale, so the effect of the data analysis is enhanced by EEMD. Undoubtedly, EEMD is a breakthrough in the development of EMD algorithm in recent years, its process is as follow:

(1) Assign the EMD algorithm running circles $N(N$ is the number of white noise added), assign the amplitude coefficient of white noise $k, n$. And execute EMD algorithm $m$ times.

(2) Add a white noise $l_{n}(t)$ to the targeted data $x(t)$,gets the noise added signal $x_{n}(t)$; Decompose the data $x_{n}(t)$ with added white noise into $I(\mathrm{IMF})_{j, n}$, where $j, n$ is the $j$-th IMF in $n$-th decomposition.

(3) Repeat (2) if $n<N$, let $n=n+1$.

(4) Obtain the means of corresponding IMFs of the decompositions as the final result. $\mathrm{C}_{j}(\mathrm{t})$ is the $j$-th IMF decomposed from the original signal by EEMD.

\subsection{CEEMD Theory}

In contract with EEMD, the decomposition process is similarly, the difference are using each noise in pairs with plus and minus signs, the final IMF is the average of the IMFs with 
positive and negative noises.

Practically, the two complementary signals can be derived by

$$
\left[\begin{array}{l}
\boldsymbol{M}_{+} \\
\boldsymbol{M}_{-}
\end{array}\right]=\left[\begin{array}{cc}
1 & 1 \\
1 & -1
\end{array}\right]\left[\begin{array}{l}
\boldsymbol{S} \\
\boldsymbol{v}
\end{array}\right]
$$

where $S$ is the original data; $v$ is the added white noise; $\boldsymbol{M}_{+}$is the sum of the original data with positive noise, and $\boldsymbol{M}_{-}$is the sum of the original data with the negative noise; $\boldsymbol{M}_{+}$and $\boldsymbol{M}_{-}$have the same amplitude, with a $180^{\circ}$ phase angle difference.

\section{Permutation Entropy}

Permutation entropy, which was introduced in [15] to measure the complexity of time series, its complexity behaves similar to Lyapunov exponents. The PE algorithm has a high sensitivity to the change of time series, which can detect the mutation of complex dynamics system.

Consider the time series $\{s(n), n=1,2, \ldots, N\}$, make phase space reconstruction for it, and get the reconstruction vector as follows:

$$
\boldsymbol{X}_{j}=[s(j), s(j+\tau), \ldots, s(j+(m-1) \tau)]
$$

Where $m$ denotes the embedding dimension, $\tau$ denotes time delay, and subscript $j=1,2, \ldots, N-(m-1) \tau . \boldsymbol{X}_{j}$ can be used as a row vector of matrix $\boldsymbol{X}$, thus each row of $\boldsymbol{X}$ can be reordered in the ascending order as

$$
s\left[j+\left(j_{1}-1\right) \tau\right] \leq s\left[j+\left(j_{2}-1\right) \tau\right] \leq \ldots \leq s\left[j+\left(j_{m}-1\right) \tau\right]
$$

Where $j_{1}, j_{2}, \ldots, j_{m}$ denote the call number of each element in the vector after phase space reconstruction. If $s\left(k+\left(j_{p}-1\right) \tau\right)=s\left(k+\left(j_{q}-1\right) \tau\right)$, then it will be ordered by the $j$ value.

Therefore, for time series $\{s(n), n=1,2, \ldots, N\}$, each row of matrix $\boldsymbol{X}$ resulting from phase space reconstruction can be assigned a set of symbol series

$$
S(g)=\left(j_{1}, j_{2}, \ldots, j_{m}\right)
$$

Where $g=1,2, \ldots, l$, hence, there are $l \leq m$ !symbol sequences will be obtained totally. The probability of each symbol sequence, $P_{1}, P_{2}, \ldots, P_{l}$ were computed, and $\sum_{g=1}^{l} P_{g}=1$.

Therefore, PE of the time series $\{s(n), n=1,2, \ldots, N\}$ is then defined as the pattern of Shannon entropy

$$
H_{p}(m)=-\sum_{g=1}^{l} P_{g} \ln P_{g}
$$

When $P_{g}=1 / m$ !, one have the maximum $\ln (m !)$, thus $H_{p}(m)$ can be normalized through $\ln (m !)$, it takes the form as

$$
H_{p}=H_{p} / \ln (m !)
$$

Obviously, $H_{p}$ are from range of 0 to1, and the value represents the randomicity of the time series. The value of $H_{p}$ is more bigger, the more random of the time series. From(2), to compute PE, both the value of the embedding dimension $m$ and time delay $\tau$ must be determined in advance. In the suggestion of literature[13], the embedding dimension $m$ 
can be range from 3 to 7 , and time delay $\tau$ can be set as 1 due to low influence to compute time series.

\section{The Elman Network Theory}

As a typically globally feed forward locally recurrent network, the Elman network [Jeffrey L. Elman et al. (1990)] contains inputs layer, the hidden layer, the network output, and the particular context layer, its expression in non-linear state space is as follows:

$$
\begin{aligned}
& y(k)=g\left(w^{3} x(k)\right) \\
& x(k)=f\left(w^{1} x_{c}(k)+w^{2}(u(k-1))\right. \\
& x_{c}(k)=x(k-1)
\end{aligned}
$$

Where $k$ stands for time, $y$ stands for $m$-dimensional vectors of output node, $x$ stands for $n$-dimensional element vector of interlayer, $u$ stands for $r$-dimensional input vectors, $x_{c}$ stands for $n$-dimensional feedback state vectors. $\omega^{3}$ stands for the connection weight between the interlayer and the output layer, $\omega^{2}$ stands for the connection weight between the input layer and the interlayer, $\omega^{1}$ stands for the connection weight between the context units layer and the interlayer. $g(\ldots)$ stands for the activation function of output neurons, $u(\ldots)$ stands for the linear combination of the output of the interlayer. $f(\ldots)$ stands for the transfer function of interneuron, generally, $s$-function are employed.

By the delay and store of the context layer, every hidden layer output is connected to every hidden unit input in this neural networks model. Therefore, this model is more activate to history data and dynamics modeling achieved, and its ability to deal with dynamics information has enhanced for interact feedback network exclusively engaged. The architecture of the Elman network is shown in Figure 1.

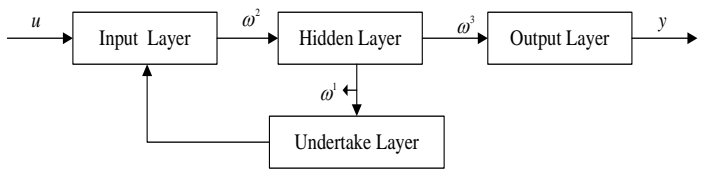

Figure 1. The Structure of Elman ANN

The weights in Elman networks can be updated by back-propagation (BP) algorithm. Error sum squares functions was taken as learning indicator function

$$
E(\omega)=\sum_{k}^{n}\left[y_{k}(\omega)-y_{k}^{*}(\omega)\right]^{2}
$$

Where $y^{*} k(\omega)$ is target output vector.

\section{Simulation of Middle-term Load Forecasting}

In this experiment, all the data applied are actual wind power of the Alberta wind farm, Canada. Those data have measured during December 1st to 27in year 2010, with a sampling interval of 10 minutes. In this paper, a experiment data set is acquired at a sampling interval of 30 minutes, previous $80 \%$ data of the data set have been considered for training and the remains $20 \%$ ( 259 samples) have been used for evaluation.

Simulations were done with ELMAN network model, which takes the form as

$$
y(t)=f_{i(t)}\left(x_{t}\right), \forall t=\Delta \ldots l
$$

Where $i(t)$ represents different time period after data decomposition, $\Delta$ represents 
embedding dimension, $\boldsymbol{x}_{t}$ contains historical wind power data.

Maximal error (ME), mean absolute percentage error (MAPE) were used as performance measures.

$\operatorname{MAPE}=100 \frac{\sum_{i=1}^{n}\left|\frac{y_{i}-\hat{y}_{i}}{y_{i}}\right|}{n}, \quad \mathrm{ME}=\max \left|y_{i}-\hat{y}_{i}\right|$

Where $y_{i}$ represents the actual wind power value, $\hat{y}_{i}$ represents the prediction value, $n$ represents the prediction sample points.

Consider the instability of the wind power time series, the original wind power time series were decomposed by CEEMD, and 200 pairs white noise sequences which are added with a standard deviation of 0.2. The IMFs decomposed are shown in Figure 2.

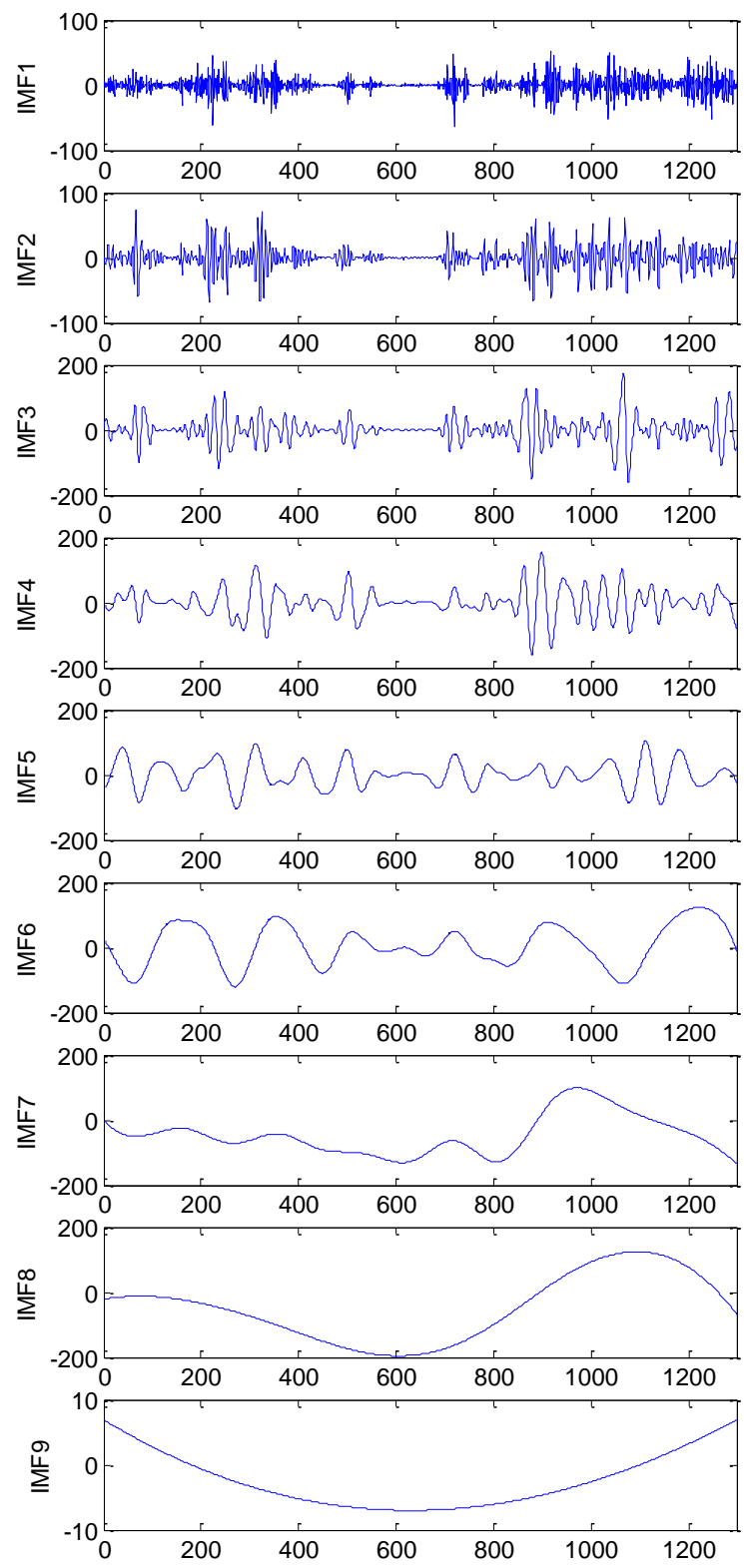




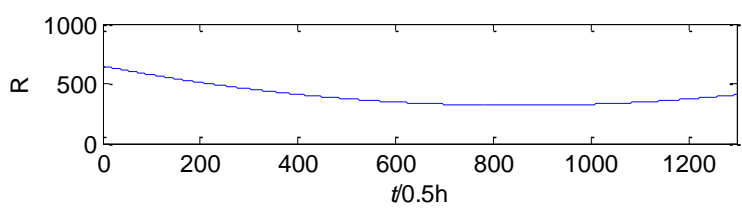

Figure 2. The Decomposed IMFs by CEEMD

Figure 3 shows the reconstruction error of EEMD and CEEMD algorithm respectively. Through Figure 3, the reconstruction errors of CEEMD are close to 0 (of the order of $10^{-12}$, determined by the calculation accuracy of computers). Whereas, due to the affects of the ensemble times with small $M$, the reconstruction errors of EEMD is much larger than those in CEEMD algorithm, which will partly affect the integrity of the decomposition.
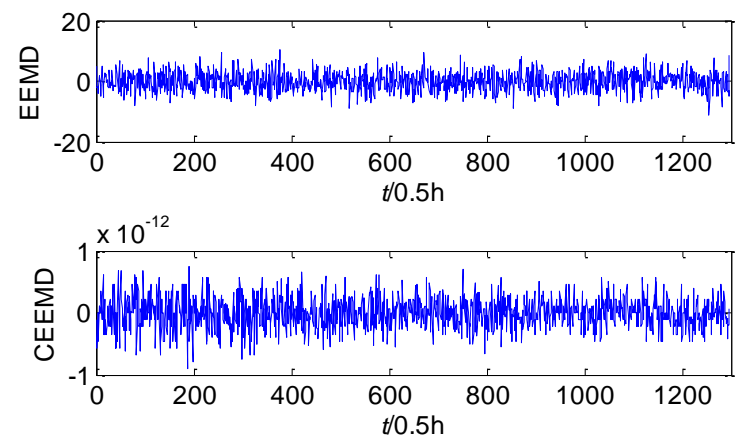

Figure 3. The Reconstruction Errors of EEMD and CEEMD (a) EEMD; (b) CEEMD

Through Figure 3, each decomposed IMFs shows strong regularity and non-stationary. However, much IMF component generated, the computation scale increased badly if the ELMAN network was used to forecast each IMF component. In order to effectively predict the wind power, PE theory was employed to analyze the complexity of each IMF component in this research. PE values of each IMF are shown in Figure 4.

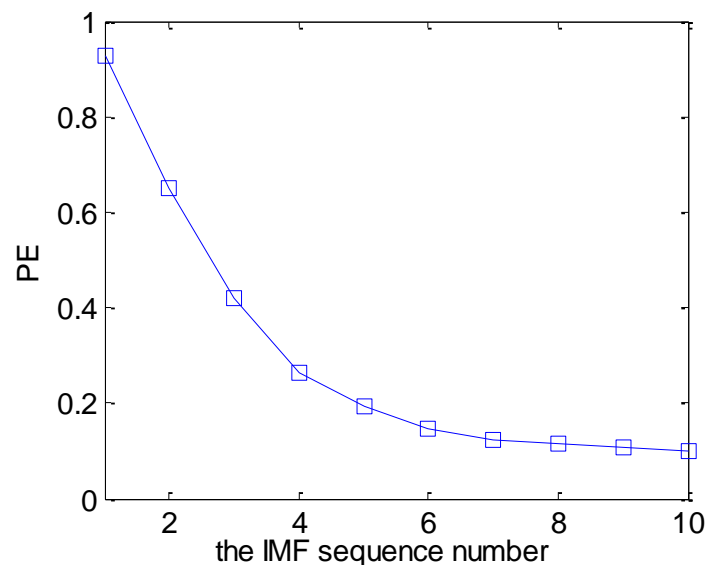

Figure 4. Permutation Entropy Values of Each IMF

As shown in Figure 4, the permutation entropy values of each IMF decrease with the reducing of frequency of IMF component, illustrate the complexity of IMF component goes down from high frequency to low frequency, thus the effectiveness of permutation entropy theory get verified. Moreover, the differences of partly neighboring permutation entropy values were little. Therefore, those IMF components can be combined to reduce 
the computation effort. The combination results are listed in Table 1. The reconstruction sequences are shown in Figure 5. When calculating PE, delay time has little effect on the results, therefore, set $\tau=1$, through the experiment, when embedding dimension $m=6$, the change rule of each IMF component is more noticeable.

\section{Table 1. The New IMF Component after Combination with PE Theory}

\begin{tabular}{c|c|c|c|c}
\hline SN of the New IMF components & F1 & F2 & F3 & F4 \\
\hline SN of the original IMF components & 1 & 2 & 3,4 & $4-10$ \\
\hline
\end{tabular}

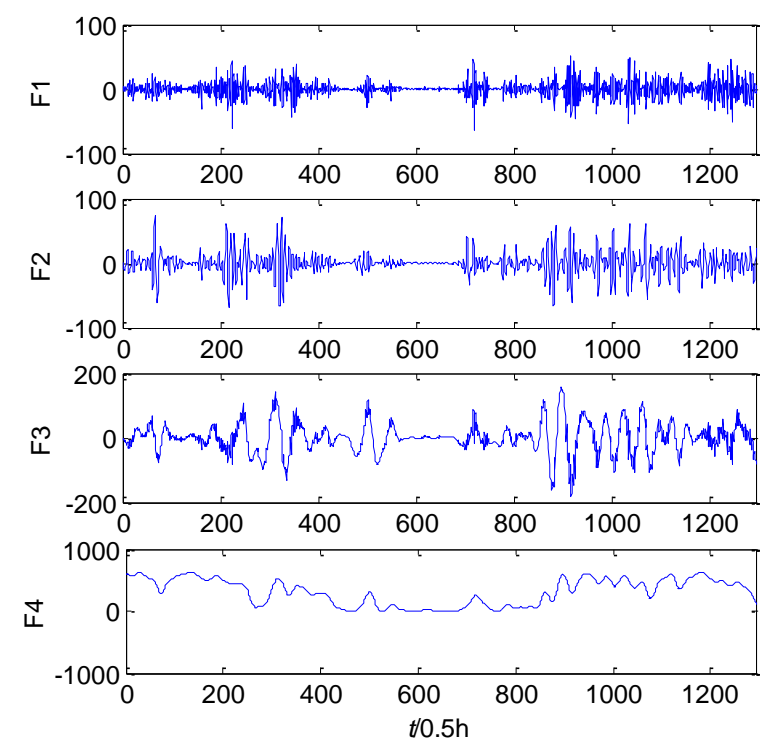

Figure 5. Recombination Component Processed By CEEMD-PE Method

In view of the four new sequences after combination, four ELMAN forecasting model were built for prediction. The steps forecasting model proposed in the paper are shown in Figure 6.

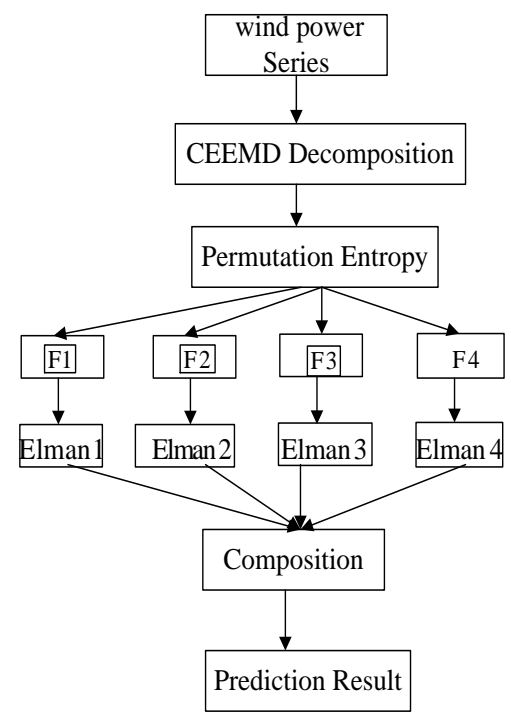

Figure 6. Combined Forecasting Flowchart Based On CEEMD-PE and ELMAN 
Then, the treated data mentioned above are inputted in the trained ELMAN numerals network to get the relative predicted value. And, the ultimate forecasting value is given by the superposition of every forecasting result. Under the same situation, the ELMAN algorithm, EEMD-Elman algorithm were used for comparison, and their forecasting effects are shown in Figure 7.Through Figure 7, it is clear that the CEEMD-PE combined with Elman achieves a better forecasting results.

In this paper, the transfer function of the intermediate layer neurons use a hyperbolic tangent transfer function, the output layer neurons is linear transfer function, the training function is Trainlm. The number of hidden layer neurons from F1-F4 is 5, 5, 10, and 3, respectively. Training steps from F1-F4 is 1000, 2000, 1000, and 500, respectively. All these parameters are obtained by cross-validation.

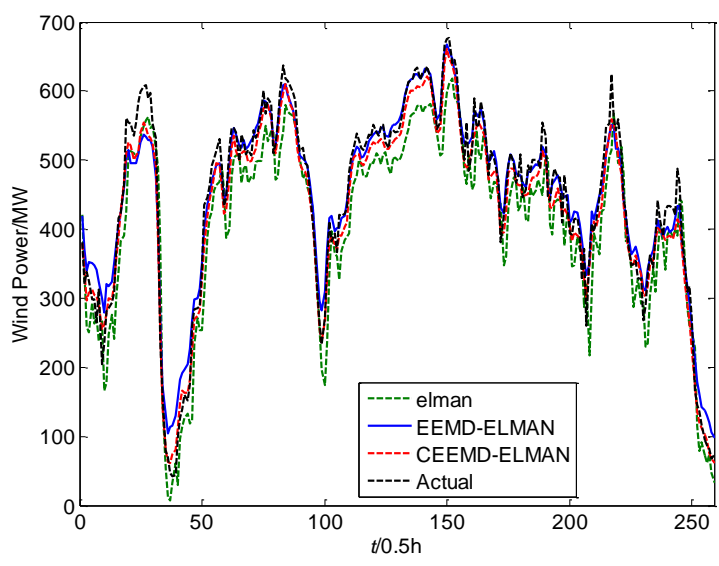

\section{Figure 7. The Forecasting Result of Different Methods}

In order to further evaluate the effectiveness of the method proposed in this paper, Table 2 demonstrates the evaluation index of MAPE and ME in the three forecasting approaches, respectively. It can be noticed that the CEEMD-Elman method has the minimum value of MAPE and ME, and the forecasting effect are better than the other two methods.

Table 2. Comparison of the Forecasting Error of the Three Models

\begin{tabular}{|l|l|l|}
\hline Model & MAPE & ME \\
\hline Elman & 12.583 & 147.497 \\
\hline EEMD-EIman & 8.8313 & 95.4419 \\
\hline CEEMD-EIman & 5.7696 & 72.8694 \\
\hline
\end{tabular}

\section{Conclusions}

A new short-term wind power prediction based on CEEMD-PE and Elman network is proposed in this paper. The wind power sequences are decomposed into a series of IMF components with different scales by CEEMD, Furthermore, the permutation entropy theory is employed in the reconstruction of IMF components to decrease the computation in the modeling of each sub-sequences. Then, the Elman forecasting models are built for each sub-sequences reconstructed, respectively. Finally, the predictive values of those components are summed to get the ultimate predictive result. The simulation results illustrate this hybrid forecasting algorithm has the best prediction precision performance, compared with Elamn algorithm and EEMD-Elamn algorithm. Thus, this method proposed has good practical value and explore a new effective way for short-term wind power prediction. 


\section{Acknowledgments}

[1]The authors would like to thank the referees for their precious reviewing. This study is fully supported by the Gansu Radio \& TV University, China (Grant No. 2014-ZD-01, Principle Investigator: Xin-you Wang).

\section{References}

[1] Costa, A. Crespo, J. Navarro, G. Lizcano, H. Madsen and E. Feitosa, "A review on the young history of the wind power short-term prediction", Renewable and Sustainable Energy reviews, vol. 12, no. 6, (2008), pp. 1725-1744.

[2] Y. S. Xue, C. Yu, J. H. Zhao, K. Li, X. Q. Liu, Q. W. Wu and G. Y. Yang, "A review on short-term and ultra-short-term wind power prediction", Automation of Electric Power Systems, vol. 39, no. 6, (2015), pp. 141-150.

[3] F. F. Gao, S. W. Wei and C. Liu, "Artificial neural network based wind power short term prediction system”, Power System Technology, vol. 32, no. 22, (2008), pp. 72-76.

[4] J. W. Zeng and W. Qiao, "Short-term wind power prediction using a wavelet support vector machine", IEEE Transactions on sustainable energy, vol. 3, no. 2, (2012), pp. 255-264.

[5] T. G. Barbounis, J. B. Theocharis, M. C. Alexiadis and P. S. Dokopoulos, "Long-Term Wind Speed and Power Forecasting Using Local Recurrent Neural network mModels", IEEE transcactions on energy Conversion, vol. 21, no. 1, (2006), pp. 273-284.

[6] N. E. Huang, Z. Shen and S. R. Long, "The empirical mode decomposition and the hilbert spectrum for nonlinear and non-stationary time series analysis", Proceedings A of the Royal Society, vol. 454, no. 1971, (1998), pp. 903-995.

[7] Z. H. Guo, W. G. Zhao, H. Y. Lu and J. Z. Wang, "Multi-step forecasting for wind speed using a modified EMD-based artificial neural network model", Renewable Energy, vol. 37, no. 1, (2012), pp. 241-249.

[8] Z. H. Zhu, Y. L. Sun and Y. Ji, "Short-term Load Forecasting Based on EMD and SVM", High Voltage Engineering, vol. 33, no. 5, (2007), pp. 118-122.

[9] Z. H. Wu and N. E. Huang, "Ensemble empirical mode decomposition: a noise-assisted data analysis method", Advances in Adaptive Data Analysis, vol. 1, no. 01, (2009), pp. 1-41.

[10] D. Liu, S. L. Pang and W. Luo, "Power system short-term load forecasting based on EEMD and dynamic neural network", Journal of Northeast DianLi University, Natural Science Edition, vol. 29, no. 6, (2009), pp. 20-26.

[11] H Wang, Z. J. Hu, Z. Chen, M. L. Zhang, J. B. He and C. Li, "A Hybrid Model for Wind Power Forecasting Based on Ensemble Empirical Mode Decomposition and Wavelet Neural Networks", Transactions of China electro technical Society, vol. 28, no. 9, (2013), pp. 137-144.

[12] X. Q. Zhang and J. Liang, "Chaotic time series prediction model of wind power based on ensemble empirical mode decomposition- approximate entropy and reservoir", Acta Physica Sinica, vol. 62, no. 5, (2013), pp. 1-10.

[13] J. R. Yeh, J. S. Shieh and N. E. Huang, "Complementary ensemble empirical mode decomposition: A novel noise enhanced data analysis method", Advances in Adaptive Data Analysis, vol. 2, no. 02, (2010), pp. $135-156$

[14] J. G. Wu and H. Lundstedt, "Prediction of geomagnetic storms from solar wind data using Elman recurrent neural networks", Geophysical research letters, vol. 23, no. 4, (1996), pp. 319-322.

[15] W. T. Chen, Z. Z. Wang, H. B. Xie and W. X. Yu, "Characterization of surface EMG signal based on Permutation Entropy", Neural Systems and Rehabilitation Engineering, IEEE Transactions on, vol. 15, no. 2, (2007), pp. 266-272. 
International Journal of Control and Automation

Vol. 10, No. 1 (2017) 